\title{
Velocity map imaging of atomic and molecular processes at the free electron laser in Hamburg (FLASH)
}

\author{
P. JOHNSSON $\dagger$, W. SIU†, A. GIJSBERTSEN $\dagger$, J. VERHOEVEN $\dagger$, \\ A.S. MEIJER $\ddagger$, W. VAN DER ZANDE $\ddagger$ and M.J.J. VRAKKING $\dagger, *$ \\ $\dagger$ FOM Institute for Atomic and Molecular Physics (AMOLF), \\ Kruislaan 407, 1098 SJ Amsterdam, The Netherlands \\ $\ddagger$ Institute for Molecules and Materials, Radboud University \\ Nijmegen, Toernooiveld 1, 6525 ED Nijmegen, The Netherlands
}

(Received Received 20 June 2008)

\begin{abstract}
Velocity map imaging was implemented at the free electron laser in Hamburg to image atomic and molecular photoionization processes at a photon energy of $45.55 \mathrm{eV}$. High quality momentum distributions were recorded for a range of rare gases ( $\mathrm{He}, \mathrm{Ne}, \mathrm{Ar}, \mathrm{Kr}$ and $\mathrm{Xe}$ ) and small molecules $\left(\mathrm{H}_{2}, \mathrm{D}_{2}, \mathrm{O}_{2}, \mathrm{~N}_{2}, \mathrm{CO}_{2}\right)$. This proof-of-principle experiment illustrates the potential for using velocity map imaging in order to study non-linear ionization and/or dissociation processes.
\end{abstract}

\section{INTRODUCTION}

The development of extreme ultraviolet (XUV)/x-ray free electron lasers (FELs), like the free electron laser in Hamburg (FLASH), offers exciting prospects for atomic and molecular physics research. Free electron lasers like FLASH offer high frequency radiation with a short pulse duration (down to 10 fs [1]) and a high flux/intensity (several tens of $\mu \mathrm{J} /$ pulse that can be focussed down to intensities of $\sim 10^{16} \mathrm{~W} \mathrm{~cm}^{-2}$ ). A tunability of FLASH down to $13.7 \mathrm{~nm}$, with harmonics extending down to $2.75 \mathrm{~nm}$, has already been demonstrated [1]. This allows for an extremely wide range of novel dynamical and structural studies. Published examples already include a first demonstration of diffractive imaging [2] and studies of atomic and molecular ionization and dissociation at high intensities [3, 4].

In this paper we present experimental results that were obtained in a campaign where a velocity map imaging spectrometer [5] was installed on the BL2 beamline of FLASH. The velocity map imaging spectrometer was placed approximately 1 meter downstream from a COLTRIMS apparatus [6] in which the FEL was brought to a focus. Thus, while being able to make use of the high photon flux of the FEL (up to $3 \times 10^{12}$ photons/pulse at a photon energy of $45.55 \mathrm{eV}$ ), the present experiments do not yet make use of the high peak intensity that can be generated. Nevertheless, the experiments are a first demonstration of the utility of velocity map imaging spectrometers at free electron lasers and pave the way for experiments involving non-linear ionization and/or dissociation, which will be attempted shortly. The velocity map imaging technique provides a $100 \%$ collection efficiency and allows for high count rates. It thus provides detection characteristics that combine very favorably with the high brilliance of FLASH.

One of the main opportunities provided by a facility like FLASH is that it readily allows to perform ionization and dissociation experiments involving non-linear photoabsorption. Ionization in strong laser fields has traditionally been investigated using low-frequency laser sources [7] where the laser frequency $\omega \ll$ the ionization potential $I_{\mathrm{p}}$ of the target atom/molecule. Ionization then proceeds by multi-photon ionization or by means of tunneling. Both involve the removal of multiple photons from the laser field. The transition between multi-photon ionization and tunneling occurs around conditions satisfying $\gamma=\left(I_{\mathrm{p}} / 2 U_{\mathrm{p}}\right)^{1 / 2} \cong 1$, where $\gamma$ is the Keldysh parameter, and where $U_{\mathrm{p}}=E_{\text {laser }}{ }^{2} / 4 \omega^{2}$ (a.u.) is the ponderomotive energy. For $\gamma \ll 1$ the ionization occurs by means of tunneling. A facility like FLASH allows to extend studies of non-linear ionization to a regime where $\omega \gg I_{\mathrm{p}}$. Under this condition significant changes in the dynamics of strong field ionization are anticipated. The $\omega^{-2}$ scaling of the ponderomotive energy suggests a greatly diminished role of field ionization in favor of a multi-photon ionization mechanism. Furthermore, stationary phase arguments dictate that the radial momentum of the electron before and after photoabsorption is the same, favoring photoabsorption at small distances of the electron from the nucleus, and hence, of inner-shell electrons.

One of the first experiments at FLASH where optical non-linearities were observed, was ionization of single xenon atoms. Wabnitz et al. used intensities of $\sim 10^{13} \mathrm{~W} \mathrm{~cm}^{-2}$ at a photon energy of $\sim 12.7 \mathrm{eV}$ and observed multiple charged ions up to $\mathrm{Xe}^{6+}$ by multiphoton sequential ionization [8]. By using a description in terms of rate equations, which take into account the simulated FEL pulses and multiphoton cross-sections, Santra et al. [9] were able to understand the high degree of ionization compared to ionization in the infrared domain. In a recent experiment, where FLASH (operating at a photon energy of $98 \mathrm{eV}$ ) was focused to intensities of $\sim 10^{16} \mathrm{~W} \mathrm{~cm}{ }^{-2}$, even higher degrees of ionization, up to $\mathrm{Xe}^{21+}$, were observed [4]. Similarly, Makris et al. [10] used rate equations to describe these observations, where the spatiotemporal structure of FLASH and multiphoton cross-sections were the necessary components. Whereas no multi-electron ejection was observed in the former experiment, it was necessary to take multiphoton single electron as well as multi-electron ejection processes into account to explain the remarkable high 
degree of ionization.

The existence of non-linearities in ionization or dissociation processes is a pre-condition for being able to study these processes in the time-domain. In time-domain pump-probe experiments, dynamical processes are commonly initiated by the absorption and/or emission of one or more pump photons, and evaluated after a given delay by means of a second interaction involving one or more probe photons. At present there is considerable excitement in the ultrafast laser community about the development of XUV attosecond pulses $[11,12]$. In the coming years investigations of electron dynamics on ultrafast time-scales will be possible [13-15], providing unique insights into the nature of photo-excitation processes, electron correlation and the interaction of electronic and nuclear degrees of freedom in photochemistry. The common technique for generating attosecond laser pulses is high harmonic generation, where atoms/molecules are exposed to an intense femtosecond laser, and where harmonics are formed by means of a three-step process consisting of ionization of the medium, acceleration of the electron in the oscillatory laser field, and emission of photons in the course of a recombination process [16, 17]. One drawback of present-day attosecond sources is that they are very weak, typical pulse energies for isolated attosecond laser pulses being on the order of 1 pJ. Free electron lasers like FLASH provide an attractive alternative, although the pulse durations achieved in high harmonic generation remain for the time being unsurpassed. Nevertheless, we consider that there exists an important complementarity between the emergence of attosecond laser pulses in table-top laboratory-scale experiments and the emergence of free electron laser facilities, where a joint user community may be able to accomplish significant progress on both sides.

A technique that has - in the last few years - become very popular in chemical physics [18] (and in attosecond science) is velocity map imaging [5]. In velocity map imaging, ions or electrons are formed at the crossing point of an atomic/molecular beam and one or more laser beams. The ions or electrons are accelerated towards a two-dimensional detector that usually consists of a dual micro-channel plate followed by a phosphor screen and a camera system. Since the extraction ion optics are designed so that the position on the camera is almost exclusively dependent on the velocity of the ion or electron, and hardly on the position where the ionization event took place. Hence, velocity map imaging measures a $2 \mathrm{D}$ projection of the $3 \mathrm{D}$ electron or ion velocity distribution that is formed in the experiment. If the experiment contains an axis of symmetry in the plane of the detector, as is usually the case, the 3D distribution can be retrieved from the 2D projection. Following the emergence of XUV attosecond pulses generated by means of high-order harmonic generation, velocity map imaging has been used to characterize the duration of attosecond pulses [19], to develop a new type of electron interferometry [20] and to observe interference in two-color attosecond $\mathrm{XUV}+$ few-cycle infrared (IR) ionization [21]. At FLASH we performed a series of proof-of-principle experiments where we used velocity map imaging to study atomic and molecular single-photon ionization processes. In this paper results for a range of small molecules will be discussed.

\section{METHODS}

\section{A. Experimental setup}

During the measurements, the laser was tuned to operate around $46 \mathrm{eV}(\lambda \sim 27 \mathrm{~nm})$ and delivered pulse trains with a repetition rate of $5 \mathrm{~Hz}$. Each train consisted of 30 micropulses spaced by $4 \mu \mathrm{s}$. The pulses had an average energy of $10 \mu \mathrm{J}$ and a pulse duration of $25 \mathrm{fs}$.

A skimmed atomic or molecular beam was formed by supersonic expansion in a pulsed gas jet operated at $5 \mathrm{~Hz}$. The target beam intersected the laser beam at right angles (see figure 1). From here we consider that the laser propagated along the $y$-axis and was polarized along the $x$-axis, while the gas jet moved along the $z$-axis. The partial pressure of the molecular beam in the interaction region was estimated to be on the order of $10^{-9} \mathrm{mbar}$, low enough to guarantee that space-charge effects are avoided. Ions or electrons created at the crossing point were accelerated towards the detector by a static electric field. The ion optics consisted of a repeller and extractor electrode. For photoelectrons an extraction field of $-1.09 \mathrm{kV} \mathrm{cm}^{-1}$ was used $\left(V_{\text {repeller }}=-9.00 \mathrm{kV}, V_{\text {extractor }}=-7.15 \mathrm{kV}\right)$ and the maximum kinetic energy that could be measured was $60 \mathrm{eV}$. For the extraction of ionic fragments, a field of typically $0.37 \mathrm{kV} \mathrm{cm}{ }^{-1}$ was used $\left(V_{\text {repeller }}=3 \mathrm{kV}, V_{\text {extractor }}=2.43 \mathrm{kV}\right)$. The maximum kinetic energy was then limited to $19 \mathrm{eV}$. The impact of the photoelectrons or ionic fragments were registered by a dual micro-channel plate/phosphor screen assembly, followed by a CCD camera. By applying a 200 ns gate to the back of the $\mathrm{MCP} /$ phosphor detector we were able to select ion masses and decrease background signals. The gate was synchronized with the last pulse in the train. In addition an on-line background subtraction was performed by measuring a background image for each second laser shot. 


\section{B. Retrieving the initial 3D angular and momentum distribution}

In our experiment the laser polarization was in the plane of the detector, providing a symmetry axis. The measured 2D projection can therefore be used in a numerical inversion procedure, to retrieve the initial 3D momentum distribution [22]. To illustrate the result of this procedure, the measured 2D projection of electrons resulting from photoionization of Neon is shown in figure $2 \mathrm{a}$ ) and a slice through the retrieved $3 \mathrm{D}$ distribution is shown in figure $2 \mathrm{~b}$ ). A sharp ring is observed at $6.7 \times 10^{-15} \mathrm{Ns}$ and corresponds to ionization to the ground state of $\mathrm{Ne}^{+}$.

A calibration of the photon energy and detector settings was performed to determine the kinetic energy of the fragments. A slice through the momentum distributions of the electrons resulting from ionization of a range of rare gases ( $\mathrm{He}, \mathrm{Ne}, \mathrm{Ar}, \mathrm{Kr}$ and $\mathrm{Xe}$ ) is shown in figure 3a-e). The electron kinetic energy spectra, resulting from an angular integration of the momentum distributions, are shown in figure 3f). In the momentum distributions of argon, krypton and xenon, several rings are observed that are attributed to inner-shell processes. The photon energy $\hbar \omega$ and the calibration of the momentum scale in the $2 \mathrm{D}$ projection, was determined by minimizing the differences between the observed and theoretical kinetic energies:

$$
\Delta=\sum_{\mathrm{He}, \mathrm{Ne}, \mathrm{Ar}, \mathrm{Kr}, \mathrm{Xe}}\left[\hbar \omega-I_{\mathrm{p}}-\frac{\text { pixels }^{2}}{\alpha}\right]^{2}
$$

$I_{\mathrm{p}}$ is the first ionization potential and $\alpha$ is a calibration parameter that provides the conversion from the measured position on the detector (in pixels ${ }^{2}$ ) to the energy of the photoelectron. During the experiments, FLASH was operated around a photon energy of $46 \mathrm{eV}$ and the photon energy that was determined by this procedure is $45.55 \pm 0.06 \mathrm{eV}$. The small standard deviation indicates excellent agreement between the measurements obtained using the different rare gases. The high accuracy is at first sight in contrast with the strong shot to shot fluctuations of FLASH [1]. The photon energy is however determined using the peak positions. Fluctuations in the photon energy will manifest themselves in the width of the peak. The mean value of the photon energy was therefore determined with an accuracy of $0.06 \mathrm{eV}$.

\section{RESULTS}

\section{A. $\mathbf{H}_{2}$ and $\mathbf{D}_{2}$}

A slice through the $3 \mathrm{D}$ momentum distribution for electrons resulting from photoionization of $\mathrm{D}_{2}$ is shown in figure 4a). In the momentum distribution a ring with maxima along the laser polarization is observed at a kinetic energy of $30.08 \pm 0.17 \mathrm{eV}$. This corresponds to ionization to the $\mathrm{D}_{2}^{+}$ground state (binding energy $15.47 \mathrm{eV}$ ).

A slice through the $3 \mathrm{D}$ momentum distribution for the $\mathrm{D}_{2}^{+}$ions is shown in figure $\left.4 \mathrm{~b}\right)$. Noting that the momentum distributions of the electrons (figure 4a)) and that of the ions are plotted on the same absolute momentum scale, figure $4 \mathrm{~b}$ ) reveals that the departure of the electron is accompanied by a matching recoil momentum of the $\mathrm{D}_{2}^{+}$. Remarkably the high fluence of FLASH allowed to observe this recoil momentum distribution within a single laser shot, while a high-quality image like figure $4 \mathrm{~b}$ ) was obtained in a few minutes.

Information about dissociative ionization processes is obtained from a slice through the $\mathrm{D}^{+} 3 \mathrm{D}$ momentum distribution, shown in figure 4c). The ion kinetic energy spectrum, resulting from angular integration of the momentum distribution, is shown in figure 4d) and shows the presence of two contributions. First, the peak at zero kinetic energy corresponds to $\mathrm{D}^{+}$ions produced by dissociative ionization to the $1 s \sigma_{\mathrm{g}}^{+}$ground state. Second a broad distribution is present for $E_{\mathrm{k}}=3.6-9 \mathrm{eV}$. These $\mathrm{D}^{+}$ions are predominantly ejected perpendicular to the laser polarization. They are attributed to a perpendicular transition to the repulsive $2 \mathrm{p} \pi_{\mathrm{u}}$ state. This state has a potential energy of $35-44 \mathrm{eV}$ in the Franck-Condon region and dissociates to $\mathrm{D}^{+}+\mathrm{D}(28.2 \mathrm{eV})$, so that $\mathrm{D}^{+}$ions formed in this dissociative ionization process are expected to have a kinetic energy of 3.4-7.9 eV. Similar results were obtained by Ito et al. [23], who observed that for a photon energy of $45 \mathrm{eV}, \mathrm{D}^{+}$ions have a broad kinetic energy distribution around $6 \mathrm{eV}$ and are mainly ejected perpendicular to the laser polarization. The results obtained for $\mathrm{H}_{2}$ are similar to $\mathrm{D}_{2}$ and are not shown here.

\section{B. $\mathrm{O}_{2}$}

A slice through the $3 \mathrm{D}$ momentum distribution of electrons resulting from photoionization of $\mathrm{O}_{2}$ is shown in figure 5a). The corresponding electron kinetic energy spectrum is shown in figure 5b). The spectrum resulting 
from an integration $\pm 5^{\circ}$ perpendicular to the laser polarization is shown in red and shows additional peaks, that were not resolved in the fully angle-integrated blue spectrum. The electron kinetic energy spectrum was calibrated by associating the highest kinetic energy peak with ionization to the $\mathrm{X}{ }^{2} \Pi_{\mathrm{g}}$ ground state and using the previously determined photon energy of $45.55 \mathrm{eV}$. The kinetic energies of the various peaks and the corresponding experimental binding energies are summarized in Table I.

The angular distribution of the data are fitted to Legendre polynomials $P_{l}$ to extract the $\beta$-parameter:

$$
P_{3 \mathrm{D}}\left(p_{3 \mathrm{D}}, \cos \theta_{3 \mathrm{D}}\right)=2 \pi \sum_{l} a_{l} P_{l}\left(\cos \theta_{3 \mathrm{D}}\right)
$$

$P_{3 \mathrm{D}}$ is the $3 \mathrm{D}$ momentum distribution. $\beta$ is the ratio of the second order and zeroth order Legendre polynomial coefficients $\left(\beta=a_{2} / a_{0}\right)$. The $\beta$ parameters are summarized in Table I. The states marked with a $\boldsymbol{\Delta}$ were only resolved in an integration perpendicular to the laser polarization and the fit included therefore neighboring states as well.

A slice through the $\mathrm{O}^{+} 3 \mathrm{D}$ momentum distribution is shown in figure $5 \mathrm{c}$ ). The corresponding ion kinetic energy spectrum is shown in figure 5d). An intense peak is present for zero kinetic energy and corresponds to the detection of $\mathrm{O}_{2}^{2+}$ ions, which did not gain a recoil velocity and/or to the formation of zero kinetic energy $\mathrm{O}^{+}$ions. Furthermore, various sharp peaks are present in the spectrum at $0.44,0.86,1.99$ and $2.94 \mathrm{eV}$. For kinetic energies up to $\sim 7 \mathrm{eV}$, a broad distribution is present.

In what follows, a discussion of the peaks in the electron kinetic energy spectrum is presented. The experimental binding energies are compared to values from literature and a tentative assignment of the peaks is performed. Possible dissociative ionization pathways of these states will be discussed in connection with the experimental ion kinetic energy spectrum.

Our data show good correspondence with the electron kinetic energy spectrum measured by Baltzer et al. [24], using $\mathrm{He} \mathrm{II} \alpha$ radiation at $40.8 \mathrm{eV}$. Below the peak at $33.24 \mathrm{eV}$, that was already assigned to the $\mathrm{X}^{2} \Pi_{\mathrm{g}}$ ground state, a relative intense peak is present for a kinetic energy of $\sim 29 \mathrm{eV}$. From Baltzer et al. [24] and Ellis et al. [25] it is known that the $\mathrm{a}^{4} \Pi_{\mathrm{u}}, \mathrm{A}^{2} \Pi_{\mathrm{u}}$ and $\mathrm{b}^{4} \Sigma_{\mathrm{g}}^{-}$states contribute in this binding energy range. Predissociation of the $\mathrm{b}^{4} \Sigma_{\mathrm{g}}^{-}$ state to the first dissociation limit at $18.73 \mathrm{eV}[26]$ can form zero kinetic $\mathrm{O}^{+}$fragments.

The peak at $E_{\mathrm{k}}=25.18 \mathrm{eV}$, in the electron kinetic energy spectrum, is assigned to the $\mathrm{B}^{2} \Sigma_{\mathrm{g}}^{-}$state. Predissociation of this state to the first dissociation limit at $18.73 \mathrm{eV}$ was reported in [26] and forms $\mathrm{O}^{+}$ions with a kinetic energy of $0.8 \mathrm{eV}$. This is in good agreement with the first intense peak in the ion kinetic energy spectrum.

In an integration perpendicular to the laser polarization axis, a small peak at $E_{\mathrm{k}}=22.60 \mathrm{eV}$ is observed. The binding energy of this state is close to the one of the $3^{2} \Pi_{u}$ state [24][25]. The deviation of our experimental value from the literature value, is attributed to the finite resolution of our spectrometer, preventing us to separate the contributions of the $\mathrm{c}^{4} \Sigma_{\mathrm{u}}^{-}$and the $3^{2} \Pi_{\mathrm{u}}$ state.

The peak at $E_{\mathrm{k}}=21.15 \mathrm{eV}$ is assigned to the $\mathrm{c}^{4} \Sigma_{\mathrm{u}}^{-}$state. This state predissociates to the first, second and fifth dissociation limit (i.e L1, L2 and L5 in Table I), forming fragments with a kinetic energy of 3,2 and $0.4 \mathrm{eV}$ respectively (see $[26,27]$ and references therein). This is in excellent agreement with the peaks at $\mathrm{E}_{\mathrm{k}}=0.44,1.99$ and $2.94 \mathrm{eV}$ in our ion kinetic energy spectrum.

The broad peak at $E_{\mathrm{k}}=12.15 \mathrm{eV}$ was assigned by Baltzer et al. [24] to the ${ }^{2} \Pi_{\mathrm{u}}$ state. Based on the theoretical threshold photoelectron spectrum, Hikosaka et al. [28] attributes this peak also to the $3^{2} \Sigma_{\mathrm{u}}^{-}$and the $5^{2} \Sigma_{\mathrm{g}}^{-}$states. Threshold photoelectron-photoion coincidence studies (TPEPICO) [28] showed that dissociation of the ${ }^{2} \Pi_{\mathrm{u}}, 3^{2} \Sigma_{\mathrm{u}}$ and $5^{2} \Sigma_{\mathrm{g}}$ states to the third, fifth and sixth dissociation limit (i.e L3, L5 and L6 in table I) produced ions with a kinetic energy of respectively 5-6 eV (L3) and $4 \mathrm{eV}$ (L5 and L6). The higher kinetic energy ions in our photoion spectrum may be due to dissociation from these states.

For binding energies higher than $36.13 \mathrm{eV}[29,30]$, single photon double ionization events should be taken into account. For the kinetic energy range $<9 \mathrm{eV}$ however, the intensity is low and only one sharp ring at $0.5 \mathrm{eV}$ is observed. It was found by Bolognesi et al. [31] that the dominant mechanism for single-photon double ionization is fast dissociation of a singly charged ion, followed by autoionization.

$$
\begin{aligned}
& \text { - } \mathrm{O}_{2}+\mathrm{hv} \rightarrow \mathrm{O}_{2}^{*+}+\mathrm{e}_{1}^{-} \\
& \text {- } \mathrm{O}_{2}^{*+} \rightarrow \mathrm{O}^{+}+\mathrm{O}^{*} \\
& \text { - } \mathrm{O}^{*} \rightarrow \mathrm{O}^{+}+\mathrm{e}_{2}^{-}
\end{aligned}
$$

The first electron $\left(\mathrm{e}_{1}^{-}\right)$has a near zero kinetic energy, while autoionization of the excited $\mathrm{O}^{*}$ leads to the formation of electrons $\left(\mathrm{e}_{2}^{-}\right.$with $E_{\mathrm{k}} \sim 0.4-2 \mathrm{eV}[32]$. 


\section{C. $\mathbf{N}_{2}$}

In figure 6a) a slice through the $3 \mathrm{D}$ momentum distribution of electrons resulting from photoionization of $\mathrm{N}_{2}$ is shown. The corresponding electron kinetic energy spectrum is shown in figure 6b). The kinetic energies of the various peaks and the corresponding binding energies are summarized in Table II. The electron kinetic energy spectrum was calibrated by associating the highest kinetic energy peak with ionization to the $\mathrm{X}^{2} \Sigma_{\mathrm{g}}^{+}$ground state and using the previously determined photon energy of $45.55 \mathrm{eV}$. The angular distributions were, when possible, fitted to extract the $\beta$ parameter (formula 2). The $\beta$ parameters are summarized in Table II as well. Based on the experimentally determined binding energies, a tentative assignment of the states can be performed and is given in Table II. The latter also contains state assignments and binding energies found in previous experiments [33, 34] and calculations [35]. Fragmentation pathways that are known from literature, are summarized in Table II, together with the expected $\mathrm{N}^{+}$kinetic energy.

A slice through the $\mathrm{N}^{+} 3 \mathrm{D}$ momentum distribution is shown in figure 6c). The corresponding ion kinetic energy spectrum is shown in figure $6 \mathrm{~d}$ ). In the ion kinetic energy spectrum, three main contributions can be distinguished. First, an intense peak at zero kinetic energy, corresponding to the intensity in the center of the image, is attributed to the detection of stable $\mathrm{N}_{2}^{2+}$ ions which did not gain a recoil velocity. Second, a peak around $1.38 \mathrm{eV}$ is associated with the formation of $\mathrm{N}^{+}$ions via dissociative ionization channels $\left(\mathrm{N}_{2} \longrightarrow \mathrm{N}^{+}+\mathrm{N}\right)$. Third, a broad asymmetric peak is present around $4 \mathrm{eV}$ and is attributed to dissociative ionization and Coulomb explosion channels. A discussion of the various states and their fragmentation pathways and products is presented.

The peak at $E_{\mathrm{k}} \sim 29 \mathrm{eV}$, in the electron kinetic energy spectrum, corresponds to ionization to the three lowest states $\mathrm{X}^{2} \Sigma_{\mathrm{g}}^{+}, \mathrm{A}^{2} \Pi_{\mathrm{u}}$ and $\mathrm{B}^{2} \Sigma_{\mathrm{u}}^{+}$. Branching ratios for these three states were already measured in the late seventies by Plummer et al. [33] and Hammet et al. [36] for a range of photon energies. For a photon energy of $45 \mathrm{eV}$ the ratios were approximately 33,55 and $12 \%$ for the $\mathrm{X}^{2} \Sigma_{\mathrm{g}}^{+}, \mathrm{A}^{2} \Pi_{\mathrm{u}}$ and $\mathrm{B}^{2} \Sigma_{\mathrm{u}}^{+}$states respectively. In our spectrum, the relative peak intensities of these states are 34, 52 and $14 \%$, in good agreement with the previous results [33, 36].

For a kinetic energy of 19.18-20.45 eV three small peaks can be distinguished in the electron kinetic energy spectrum. In this energy range Baltzer et al. [34] reported the presence of three states of which two states $\left(2^{2} \Pi_{\mathrm{g}}\right.$ and $\mathrm{C}^{2} \Sigma_{\mathrm{u}}^{+}$ states) exhibited vibrational progressions.

In the electron kinetic energy spectrum, a relatively intense peak can be identified at $E_{\mathrm{k}}=16.38 \mathrm{eV}$ and corresponds to the $\mathrm{F}^{2} \Sigma_{\mathrm{g}}^{+}$state. Fragmentation to the second and third dissociation limit (i.e L2 and L3 in Table II) has been reported in $[35,37]$. In the $\mathrm{N}^{+}$kinetic energy spectrum a broad peak is present at $1.35 \mathrm{eV}$ and is predominantly attributed to dissociation of the $\mathrm{F}^{2} \Sigma_{\mathrm{g}}^{+}$state to the former (i.e L2) dissociation limit.

A relatively weak contribution is present for $E_{\mathrm{k}}=11-14 \mathrm{eV}$ in the electron kinetic energy spectrum. Fragments produced from dissociation of this state, are expected to have a kinetic energy of 2.66-3.16 eV.

The last peak in the electron kinetic energy spectrum $\left(E_{\mathrm{k}}=7.71 \mathrm{eV}\right)$ is associated with transitions to the $5^{2} \Sigma_{\mathrm{g}}^{+}$and $6^{2} \Sigma_{\mathrm{g}}^{+}$states [35]. The potential energy curves of these states correlate adiabatically to the sixth dissociation limit (i.e L6 in Table II) at $28.757 \mathrm{eV}$ [35]. Fragmentation via this pathway produces fragments with $\mathrm{E}_{\mathrm{k}}=4.7 \mathrm{eV}$. Non-adiabatic transitions to the third and seventh dissociation limits (L3 and L7 in Table II) were identified by Aoto et al. [35] and produce fragments with a kinetic energy of 5.7 and $4.1 \mathrm{eV}$ respectively. All these features are consistent with the broad kinetic energy distribution extending from 2 to $7 \mathrm{eV}$ as observed in the measurements.

In addition to dissociative ionization channels, Coulomb explosion channels also play a role for this photon energy. Coulomb explosion channels were studied by Besnard et al. [38] using photoion-photoion coincidence spectroscopy (PIPICO) in the range of $45-60 \mathrm{eV}$. For a photon energy of $45-46 \mathrm{eV}$, the $\mathrm{N}_{2}^{2+}{ }^{1} \Pi_{\mathrm{u}}$ (the $\alpha$ state) and the ${ }^{3} \Sigma_{\mathrm{g}}^{-}$(the $\beta$ state) with vertical binding energies of $45.2 \pm 0.5 \mathrm{eV}$ and $46.1 \pm 0.3 \mathrm{eV}$, should be considered. Dissociation to the first fragmentation threshold $\mathrm{N}^{+}\left({ }^{3} \mathrm{P}\right)+\mathrm{N}^{+}\left({ }^{3} \mathrm{P}\right)$ at $38.8 \mathrm{eV}$ forms $\mathrm{N}^{+}$ions with a kinetic energy of $3.2 \pm 0.3 \mathrm{eV}$ and $3.7 \pm 0.2 \mathrm{eV}$. These kinetic energies fall into the most intense part of the broad asymmetric peak.

\section{D. $\mathrm{CO}_{2}$}

A slice through the $3 \mathrm{D}$ momentum distribution of electrons resulting from photoionization of $\mathrm{CO}_{2}$ is shown in figure 7a). The corresponding electron kinetic energy spectrum is shown in figure 7b). The photoelectron spectrum was calibrated by associating the highest kinetic energy peak with ionization to the $\mathrm{X}^{2} \Pi_{\mathrm{g}}^{+}$ground state and using the previously determined photon energy of $45.55 \mathrm{eV}$. The kinetic energies of the various peaks and the corresponding binding energies are summarized in Table III. Based on the experimental binding energies a tentative assignment of the states can be performed. The binding energies of the assigned states as found in previous experiments [39] are summarized in Table III. The $\beta$ parameters were, when possible, extracted from the data and are listed in Table III as well. 
A slice through the $\mathrm{CO}^{+}$and $\mathrm{O}^{+} 3 \mathrm{D}$ momentum distribution is shown in figure $7 \mathrm{c}$ ) and e) respectively. The corresponding ion kinetic energy spectra are shown in figure $7 \mathrm{~d}$ ) and f). In the kinetic energy spectra of $\mathrm{CO}^{+}$and $\mathrm{O}^{+}$two main features can be distinguished, namely intense peaks at zero kinetic energy and broad asymmetric distributions at higher kinetic energies that are suggestive of multiple contributions. The distributions of $\mathrm{CO}^{+}$and $\mathrm{O}^{+}$ions seem to be momentum matched, suggesting that they may arise through a common fragmentation pathway. A slice through the $3 \mathrm{D}$ momentum distribution of the $\mathrm{C}^{+}$ions is shown in figure $7 \mathrm{~g}$ ). The corresponding kinetic energy spectrum is shown in figure $7 \mathrm{~h}$ ). In the spectrum an intense peak at zero kinetic energy is present, as well as a broad asymmetric distribution with an energy up to $3 \mathrm{eV}$.

In what follows a discussion of the assigned states in the electron kinetic energy spectrum and possible fragmentation pathways from the assigned states is given.

In the electron kinetic energy spectrum, the peak at $E_{\mathrm{k}}=31.79 \mathrm{eV}$ is associated with ionization of an essentially non-bonding electron localized on an $\mathrm{O}$ atom and corresponds to ionization to the $\mathrm{X}^{2} \Pi_{\mathrm{g}}^{+}$state.

The next peak is observed at $E_{\mathrm{k}}=27.74 \mathrm{eV}$. From previous experiments [40,41] it is known that two states contribute in this binding energy region, namely the $\mathrm{A}^{2} \Pi_{\mathrm{u}}^{+}$and $B^{2} \Sigma_{\mathrm{u}}^{+}$states. Due to vibrational excitation these peaks overlap and are not resolved in our experiment.

From an integration $\pm 5^{\circ}$ along the laser polarization axis (shown in red in figure $7 \mathrm{~b}$ )), an asymmetric feature on the left side of the second peak can be distinguished at $E_{\mathrm{k}}=26.31 \mathrm{eV}$. This peak is associated with ionization to the $\mathrm{C}^{2} \Sigma_{\mathrm{g}}^{+}$state. It was found that this state predissociates to $\mathrm{O}^{+}\left({ }^{4} \mathrm{~S}_{\mathrm{u}}\right)+\mathrm{CO}\left(\mathrm{X}^{1} \Sigma^{+}\right)$at $19.07 \mathrm{eV}[42]$ and to $\mathrm{CO}^{+}\left(\mathrm{X}^{2} \Sigma^{+}\right)+\mathrm{O}\left({ }^{3} \mathrm{P}\right)$ at $19.465 \mathrm{eV}$. Fragmentation via these pathways produces near zero kinetic energy $\mathrm{O}^{+}$ions $[42,43]$ and $\mathrm{CO}^{+}$ions [42].

For lower kinetic energies, various peaks are observed that were previously associated with multi-electron transitions (MET) $[44,45]$. The binding energies of these states from Brion et al. [44] are listed in Table III. A dissociative electroionization study by Locht et al. [46] identified a series of fragmentation pathways, producing $\mathrm{C}^{+}, \mathrm{O}^{+}$and $\mathrm{CO}^{+}$, for a couple of these MET states. Relevant pathways are shown in Table III together with the measured kinetic energies of the ionic fragments by Locht et al. Our measured kinetic energies for the $\mathrm{C}^{+}$ion are in good agreement with these results.

The threshold for single-photon double ionization is at $37.34 \pm 0.01 \mathrm{eV}$ [47]. The threshold value for the formation of $\mathrm{CO}^{+}$and $\mathrm{O}^{+}$ions was found to be $39.2 \pm 0.3 \mathrm{eV}$ [48]. For this energy, two states should be considered: $\mathrm{X}^{3} \Sigma_{\mathrm{g}}^{-}$ and $\mathrm{a}^{1} \Delta_{\mathrm{g}}$. Fragmentation pathways of these states together with the expected kinetic energies of the fragment ions, are summarized in Table IV.

The kinetic energy distribution of the $\mathrm{CO}^{+}$ions in figure $7 \mathrm{~d}$ ) is centered around $2.3 \mathrm{eV}$. Its asymmetric shape is suggestive of multiple contributions, with a small maximum at $1.5 \mathrm{eV}$. In the $\mathrm{O}^{+}$ion kinetic energy spectrum (figure 7f)), two maxima are observed at 2.3 and $3.7 \mathrm{eV}$. Comparison with the kinetic energy values listed in Table IV suggests that dissociation to the first limit, forming $\mathrm{CO}^{+}$ions with an energy of $2.2 \mathrm{eV}$ and $\mathrm{O}^{+}$ions with an energy of $3.9 \mathrm{eV}$ is an important contribution. Our kinetic energy release of $6 \mathrm{eV}$ is in good agreement with the results from Curtis et al. [49]. In addition, dissociation to the third limit is in good agreement with the maximum at $2.3 \mathrm{eV}$ in the $\mathrm{O}^{+}$photoion spectrum and the feature at $1.5 \mathrm{eV}$ in the $\mathrm{CO}^{+}$photoion spectrum.

We note that, although the agreement suggested by the analysis presented above is quite good, additional electronic states exist between the $39.2 \mathrm{eV}$ threshold and our $45.55 \mathrm{eV}$ photon energy, which could have contributed to our experiment. Furthermore the importance of contribution from dissociative ionization in the relative kinetic energy range cannot be ruled out.

\section{DISCUSSION}

In this paper we have reported on experiments where the velocity map imaging technique was for the first time implemented at the free electron laser in Hamburg. We have imaged single-photon ionization processes of a range of small molecules $\left(\mathrm{H}_{2}, \mathrm{D}_{2}, \mathrm{O}_{2}, \mathrm{~N}_{2}\right.$ and $\left.\mathrm{CO}_{2}\right)$, allowing us to test the capabilities of the velocity map imaging technique. Our results demonstrate both the strengths and weaknesses of the implemented spectrometer in combination with FLASH.

In the discussion of the results, it became evident that the resolution of our spectrometer was limiting (typically 1$2 \%$ ), compared to i.e time-of-flight spectrometers or electrostatic analyzers. However, while these techniques provide superior energy resolution over the velocity map imaging technique, they lack angular resolution. Previous high intensity IR experiments have shown that the angular distribution contains a large part of the information about non-linear ionization processes and are essential in the interpretation of high-intensity experiments.

The interpretation of dissociative ionization processes can be complicated when several states contribute in the same kinetic energy range. Indeed definite fragmentation pathways could not always be identified. A commonly used technique to study dissociation processes is COLTRIMS. While providing coincidence, this technique is limited to low 
count rates, making it a challenging technique in combination with the low repetition rate of FLASH. The velocity map imaging technique allows for high count rates and thus makes optimal use of the high brilliance and intensity of FLASH.

A type of experiments that makes use of the high fluence and the time-resolution of the FLASH are pump-probe experiments. These experiments provide a wealth of information about the dynamics of photoexcitation processes, but require that measurements at a given pump-probe delay are completed in a short time. For example, FLASH can be used to dissociatively ionize molecules that have been dynamically aligned by an intense femtosecond IR laser pulse. By synchronizing FLASH with the time of an alignment revival, angle-resolved photoelectron momentum distributions can be obtained from aligned molecules. These distributions provide information about the photoemission process in the molecular frame, not measured before due to the intensity of the current XUV sources. Dynamic alignment has been successfully probed by velocity map imaging in the past [50]. Velocity map imaging is thus a promising technique for studying dynamic alignment, as well as (dissociative) ionization processes at FLASH, providing accessibility to high count rates and angular resolution.

\section{ACKNOWLEDGEMENTS}

This work is part of the research program of the "Stichting voor Fundamenteel Onderzoek der Materie (FOM)", which is financially supported by the "Nederlandse organisatie voor Wetenschappelijk Onderzoek (NWO)". Financial support by the Marie Curie Research Training Networks "XTRA" is gratefully acknowledged. P.J. would like to acknowledge the support of the Swedish Science Council. In addition, we would like to thank the staff of the FLASH FEL in Hamburg, in particular Michael Gensch and Rolf Treusch, as well as Joachim Ullrich, Robert Moshammer, Artem Rudenko and Claus Dieter Schröter of the Max-Planck Institut für Kernfysik in Heidelberg. Rob Kemper is acknowledged for his invaluable role in preparing the experimental apparatus, and Ad de Snaijer and Hinco Schoenmaker for their assistance with the on-site installation in Hamburg.

[1] Ackermann, W.; Asova, G.; Ayvazyan, V.; Azima, A.; Baboi, N.; Bähr, J.; Balandin, V.; Beutner, B.; Brandt, A.; et al. Operation of a free-electron laser from the extreme ultraviolet to the water window. Nature Photonics 2007, 1, 336.

[2] Chapman, H.N.; Barty, A.; Bogan, M.J.; Boutet, S.; Frank, M.; Hau-Riege, S.P.; Marchesini, S.; Woods, B.W.; Bajt, S.; et al. Femtosecond diffractive imaging with a soft-X-ray free-electron laser. Nature Phys. 2006, $2,839$.

[3] Sorokin, A.A.; Bobashev, S.V.; Tiedtke, K.; et al. Multi-photon ionization of molecular nitrogen by femtosecond soft x-ray FEL pulses. J. Physics B: At. Mol. Opt. Phys. 2006, 39, L299.

[4] Sorokin, A.A.; Bobashev, S.V.; Feigl, T.; Tiedtke, K.; Wabnitz, H.; et al. Photoelectric effect at ultrahigh intensities. Phys. Rev. Lett. 2007, 99, 213001.

[5] Eppink, A.T.J.B.; Parker, D.H. Velocity map imaging of ions and electrons using electrostatic lenses: Application in photoelectron and photofragment ion imaging of molecular oxygen. Rev. Sci. Instrum. 1997, 68, 3477.

[6] Mergel, V.; Dörner, R.; Ullrich, J.; Jagutzki, O.; Lencinas, S.; Nüttgens, S.; Spielberger, L.; Unverzagt, M.; Cocke, C.L.; et al. $\mathrm{He}^{2+}$ on He: State-selective, scattering-angle-dependent capture cross sections measured by cold target recoil ion momentum spectroscopy (COLTRIMS). Nucl. Instrum. Methods Phys. Res. B 1995, 98, 593.

[7] Brabec, T.; Krausz, F. Intense few-cycle laser fields: Frontiers of nonlinear optics. Rev. Mod. Phys. $2000,72,545$.

[8] Wabnitz, H.; de Castro, A.R.B.; Gürtler, P.; Laarmann, T.; Laasch, W.; Schulz, J.; et al. Multiple Ionization of Rare Gas Atoms Irradiated with Intense VUV Radiation. Phys. Rev. Lett. 2005, 94, 023001.

[9] Santra, R.; Greene, C.H. Multiphoton ionization of xenon in the vuv regime. Phys. Rev. A 2004, 70, 053401.

[10] Makris, M.G.; Lambropoulos, P.; Mihelič, (Private communication)

[11] Paul, P.M.; Toma, E.S.; Breger, P.; Mullot, G.; Augé, F.; Balcou, P.; Muller, H.G.; et al. Observation of a Train of Attosecond Pulses from High Harmonic Generation. Science 2001, 292, 1689.

[12] Hentschel, M.; Kienberger, R.; Spielmann, C.; Reider, G.A.; Milosevic, N.; Brabec, T.; Corkum, P.; Heinzmann, U.; Drescher, M.; et al. Attosecond metrology. Nature 2001, 414, 509.

[13] Drescher, M.; Hentschel, M.; Kienberger, R.; Tempea, G.; Spielmann, C.; Reider, G.A.; Corkum, P.B.; et al. X-Ray Pulses Approaching the Attosecond Frontier. Science 2001, 291, 1923.

[14] Uiberacker, M.; Uphues, T.; Schultze, M.; Verhoef, A.J.; Yakovlev, V.; Kling, M.F.; Rauschenberger, J.; Kabachnik, N.M.; Schröder, H.; et al. Attosecond real-time observation of electron tunnelling in atoms. Nature 2007, 446, 627.

[15] Cavalieri, A.L.; Müller, N.; Uphues, T.; Yakovlev, V.S.; Baltuška, A.; Horvath, B.; Schmidt, B.; Blümel, L.; Holzwarth, R.; et al. Attosecond spectroscopy in condensed matter. Nature 2007, 449, 1029.

[16] Schafer, K.J.; Yang, B.; DiMauro, L.F.; et al. Above threshold ionization beyond the high harmonic cutoff. Phys. Rev. Lett. 1993, 70, 1599.

[17] Corkum, P.B. Plasma perspective on strong-field multiphoton ionization. Phys. Rev. Lett. $1993,71$.

[18] Whitaker, B. Imaging in Molecular Dynamics: Technology and Applications.; Cambridge University Press, 2003. 
[19] Aseyev, S.A.; Ni, Y.; Frasinski, L.J.; Muller, H.G.; et al. Attosecond Angle-Resolved Photoelectron Spectroscopy. Phys. Rev. Lett. 2003, 91, 223902.

[20] Remetter, T.; Johnsson, P.; Mauritsson, J.; Varjú, K.; Ni, Y.; Lépine, F.; Gustafsson, E.; Kling, M.; Khan, J.; et al. Attosecond Electron Wave Packet Interferometry. Nature Phys. 2006, 2, 323.

[21] Mauritsson, J.; Remetter, T.; Swoboda, M.; Klünder, K.; L'Huillier, A.; Schafer, K.J.; Kelkensberg, F.; Siu, W.; Ghafur, O.; et al. in preparation

[22] Vrakking, M.J.J. An iterative procedure for the inversion of two-dimensional ion/photoelectron imaging experiments. Rev. Sci. Intrum. 2001, 72, 4084.

[23] Ito, K.; Hall, R.I.; Ukai, M. Dissociative photoionization of $\mathrm{H}_{2}$ and D2 in the energy region of 2545 eV. J. Chem. Phys. 1996, 104, 8449.

[24] Baltzer, P.; Wannberg, B.; Karlsson, L.; Carlsson Göthe, M.; et al. High-resolution inner-valence uv photoelectron spectra of the $\mathrm{O}_{2}$ molecule and configuration-interaction calculations of ${ }^{2} \Pi_{\mathrm{u}}$ states between 20 and 26 eV. Phys.Rev. A 1992, 45, 4374.

[25] Ellis, K.; Hall, R.I.; Avaldi, L.; Dawber, G.; McConkey, A.; Andric, L.; et al. High resolution threshold photoelectron and photoion spectroscopy of oxygen in the 12-50 eV photon range. J. Phys. B: At. Mol. Opt. Phys 1994, $27,3415$.

[26] Richard-Viard, M.; Dutuit, O.; Lavollée, M.; Govers, T.; Guyon, P.M.; et al. $\mathrm{O}^{+}$ions dissociation studied by threshold photoelectronphotoion coincidence method. J. Chem. Phys 1985, 82, 4054.

[27] Lu, Y.; He, Z.X.; Cutler, J.N.; Southworth, S.H.; Stolte, W.C.; et al. Dissociative photoionization study of $\mathrm{O}_{2}$. J Electron spectrosc. Relat. Phenom. 1998, 94, 135-147.

[28] Hikosaka, Y.; Aoto, T.; Hall, R.I.; Ito, K.; Hirayama, R.; Yamamoto, N.; et al. Inner-valence states of $\mathrm{O}_{2}^{+}$and dissociation dynamics studied by threshold photoelectron spectroscopy and a configuration interaction calculation. J. Chem. Phys. 2003, 119,7693

[29] Pettersson, L.G.M.; Larsson, M. On the character of the $\mathrm{O}_{2}^{2+} \mathrm{A}^{3} \Sigma_{\mathrm{u}}^{+}$state. J. Chem. Phys. 1991, $94,818$.

[30] Hall, R.I.; Dawver, G.; McConkey, A.; MacDonald, M.A.; et al. Vibrational structure of the $\mathrm{O}_{2}^{2}+$ ground state observed by threshold photoelectron coincidence spectroscopy. Phys. Rev. Lett. 1992, 68, 2751.

[31] Bolognesi, P.; Thompson, D.B.; Avaldi, L.; MacDonald, M.A.; Lopes, M.C.A.; Cooper, D.R.; et al. Vibrationally selected $\mathrm{O}^{+}-\mathrm{O}^{+}$fragmentation of $\mathrm{O}_{2}$ below the adiabtic double-ionization potential studied via electron-electron coincidence spectroscopy. Phys. Rev. Lett. 1999, 82, 2075.

[32] Feifel, R.; Eland, J.H.D.; Edvardsson, D. Valence double ionization of $\mathrm{O}_{2}$ at photon energies below and above the molecular double ionization threshold. J. Chem. Phys. 2005, 122, 144308.

[33] Plummer, E.W.; Gustafsson, T.; Gudat, W.; et al. Partial photoionization cross sections of $\mathrm{N}_{2}$ and CO using synchrotron radiation. Phys. Rev. A 1977, 15, 2339.

[34] Baltzer, P.; Larsson, M.; Karlsson, L.; Wannberg, B.; et al. Inner-valence states of $\mathrm{N}_{2}^{+}$studied by uv photoelectron spectroscopy and configuration-interaction calculations. Phys.Rev. A 1992, 46, 5545.

[35] Aoto, T.; Ito, K.; Hikosaka, Y.; Shibasaki, A.; Hirayama, R.; Yamamono, N.; et al. Inner-valence states of $\mathrm{N}_{2}^{2+}$ and the dissociation dynamics studied by threshold photoelectron spectroscopy and configuration interaction calculation. J. Chem. Phys. 2006, 124, 234306.

[36] Hammet, A.; Stoll, W.; Brion, C. Ibid 1976, 8, 367.

[37] Eland, J.H.D.; Duerr, E.J. Dissociation and electronion angular distributions in inner-valence photoionisation of CO and $\mathrm{N}_{2}$. Chem. Phys 1998, 229, 13.

[38] Besnard, M.J.; Hellner, L.; Dujardin, G.; et al. Photoion-Photoion coincidence study of the fragmentation of the double charged $\mathrm{N}_{2}^{2+}$ cation. J. Chem. Phys. 1988, 88, 1732 .

[39] Turner, D.W.; May, D.P. Franck-Condon factors in ionization: experimental measurement using molecular photoelectron spectroscopy.II. J. Chem. Phys. 1967, 46, 1156.

[40] Kovač, B. The HeI photoelectron spectra of $\mathrm{CO}_{2}, \mathrm{CS}_{2}$ and OCS: vibronic coupling. J. Chem. Phys. $1983,78,1684$.

[41] Wang, L.S.; Reutt, J.E.; Lee, Y.T.; et al. High resolutuion UV spectroscopy of $\mathrm{CO}_{2}^{+}$and $\mathrm{CS}_{2}^{+}$using supersonic molecular beams. J Electron spectrosc. Relat. Phenom. 1988, 47, 167.

[42] Bombach, R.; Dannacher, J.; Stadelmann, J.P.; et al. Branching ratios and partition of the excess energy for the predissociation of $\mathrm{CO}_{2}^{+} \mathrm{C}^{2} \Sigma_{g}^{+}$molecular cations. J. Chem. Phys. 1983, 79.

[43] Liu, J.; Chen, W.; Hochlaf, M.; Qian, X.; Chang, C.; et al. Unimolecular decay pathways of state-selected $\mathrm{CO}_{2}^{+}$in the internal energy range of 5.2-6.2 eB: an experimental and theoretical study. J. Chem. Phys 2003, 118, 149.

[44] Brion, C.E.; Tan, K.H. Partial oscillator strengths for the photoionization of $\mathrm{N}_{2} \mathrm{O}$ and $\mathrm{CO}_{2}(20-60 \mathrm{eV})$. Chem. Phys. 1978, 34, 141.

[45] Potts, A.W.; Williams, T.A. The observation of forbidden transistions in the He II photoelectron spectra. J. Electron spectrosc. Relat. Phenom. 1974, 3, 3.

[46] Locht, R.; Davister, M. The dissociative electroionization of carbon dioxide by low-energy electron impact. The $\mathrm{C}^{+}, \mathrm{O}^{+}$ and $\mathrm{CO}^{+}$dissociation channels. Int. J. Mass Spectrom. Ion Process. 1995, 144, 105.

[47] Slattery, A.E.; Field, T.A.; Ahmad, M.; Hall, R.I.; Lambourne, J.; Penent, F.; Lablanquie, P.; et al. Spectroscopy and metastability of $\mathrm{CO}_{2}^{2+}$ molecular ions. J. Chem. Phys 2005, 122, 084317.

[48] Masuoka, T.; Nakamura, E.; Hiraya, A. Kinetic-energy release in the dissociation of $\mathrm{CO}_{2}^{2}+$. J. Chem. Phys. 1996, 104, 6200.

[49] Curtis, D.M.; Eland, J.H.D. Coincidence studies of doubly charged ions formed by 30.4 nm photoionization. Int. J. Mass Spectrom. Ion Process. 1985, 63, 241. 
[50] Rosca-Pruna, F.; Vrakking, M.J.J. Experimental observation of revival structures in picosecond laser-induced alignment of $\mathrm{I}_{2}$. Phys. Rev. Lett. 2001, 87, 153902.

[51] Akahori, T.; Morioka, Y.; Watanabe, M.; Hayaishi, T.; Ito, K.; et al. Dissociation processes of $\mathrm{O}_{2}$ in the VUV region 500-700 AA. J. Phys. B 1985, 18, 2219.

[52] Nicolas, C.; Alcaraz, C.; Thissen, R.; Vervloet, M.; et al. Dissociative photoionization of $\mathrm{N}_{2}$ in the $2432 \mathrm{eV}$ photon energy range. J. Phys. B: At. Mol. Opt. Phys. 2003, 36, 2239. 


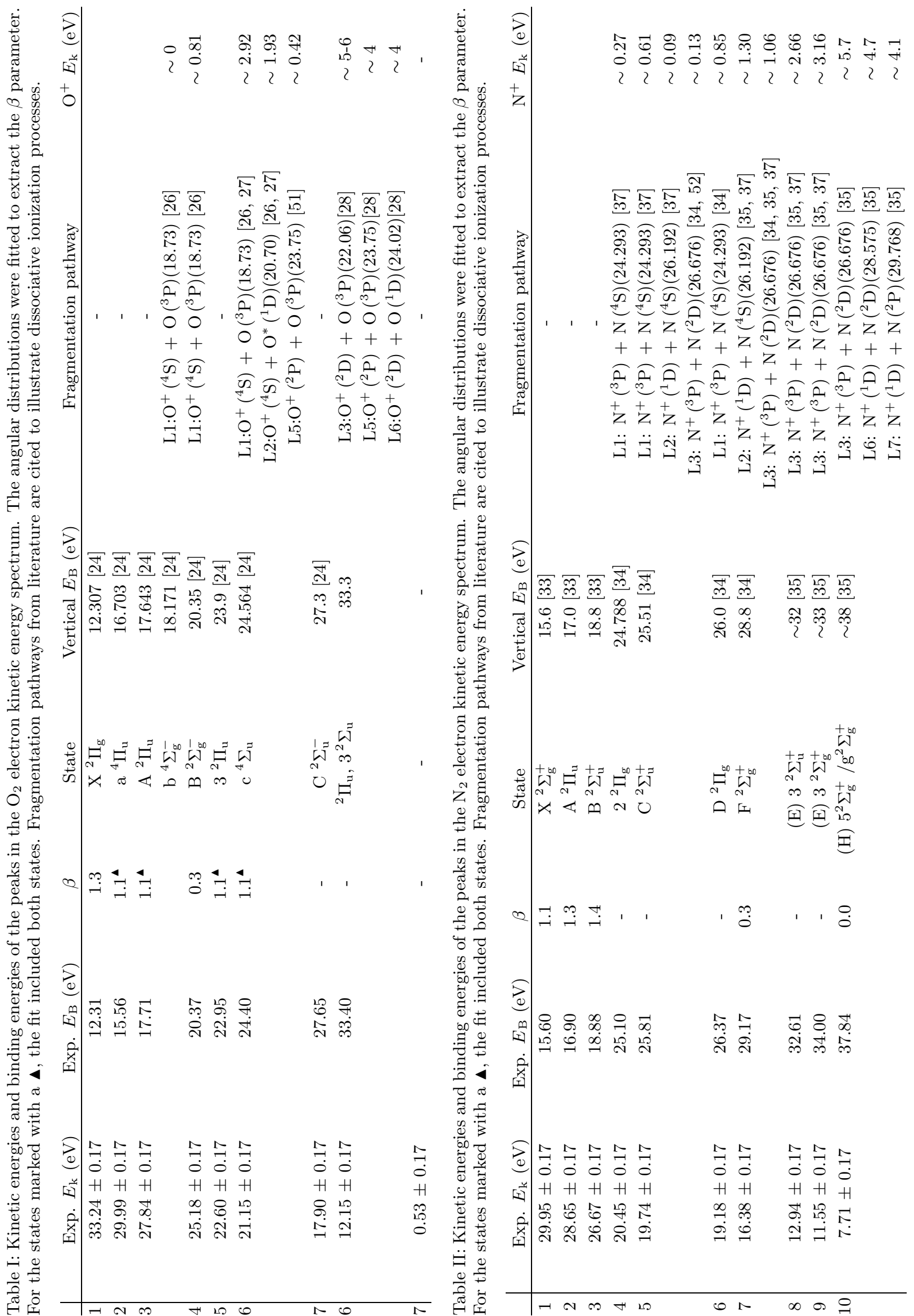




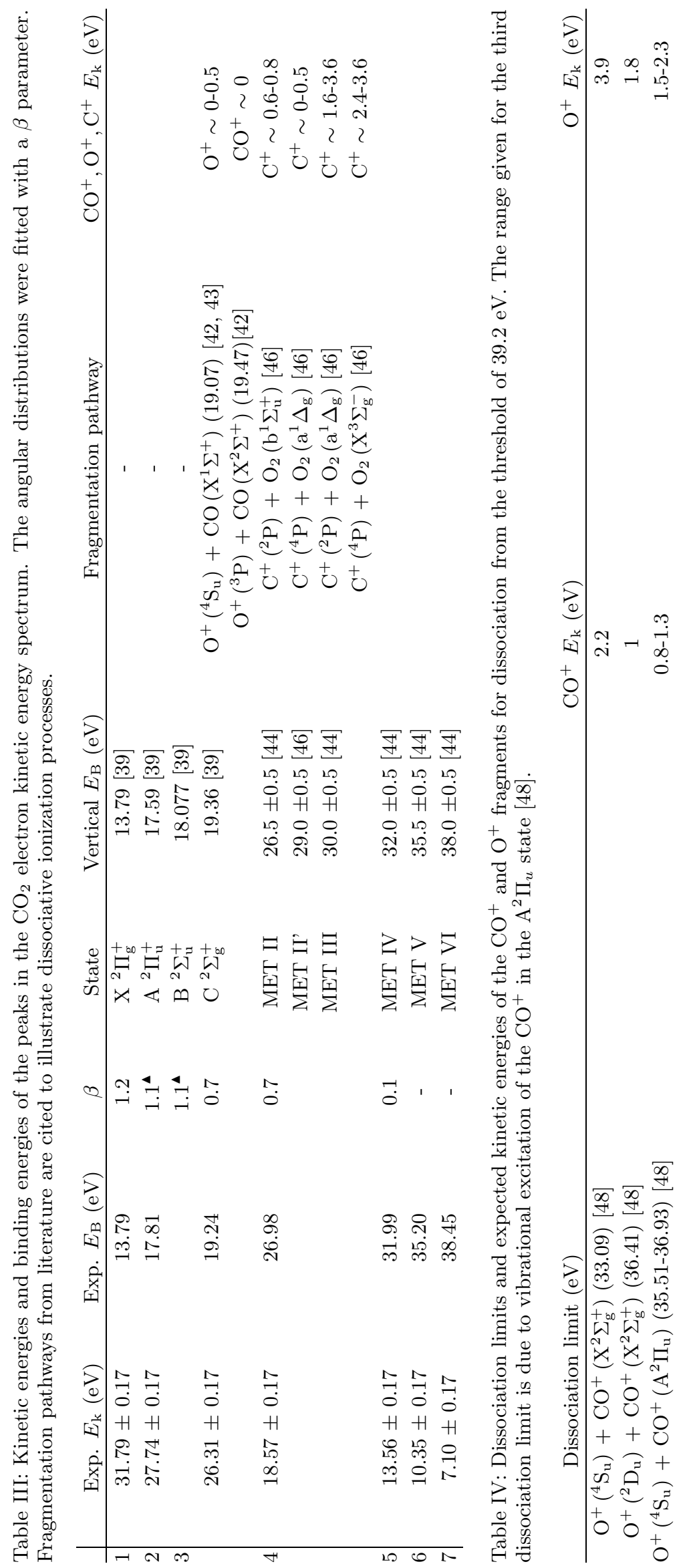


Figure 1. A schematic illustration of the experimental set-up. The focus of FLASH was approximately $1 \mathrm{~m}$ before the center of the interaction region. Photoelectrons or ionic fragments formed at the crossing point of the laser beam and the molecular beam were accelerated by the static electric field towards a dual MCP/phosphor screen assembly, where their impact was registered by a CCD camera.

Figure 2. a) The measured projection of the momentum distribution of electrons resulting from photoionization of Neon. b) A slice $\left(p_{z}=0\right)$ through the retrieved 3D momentum distribution from the measured projection.

Figure 3. a) A slice $\left(p_{z}=0\right)$ through the 3D momentum distribution of electrons resulting from ionization of a) Helium, b) Neon, c) Argon, d) Krypton and e) Xenon. f) The electron kinetic energy spectra resulting from angular integration of the 3D momentum distributions.

Figure 4. a) A slice $\left(p_{z}=0\right)$ through the retrieved $3 \mathrm{D}$ momentum distribution electrons resulting from ionization of $\mathrm{D}_{2}$. The momentum distribution is peaked along the laser polarization and is maximum for $E_{\mathrm{k}}=30.08 \pm$ $0.17 \mathrm{eV}$. b) A slice $\left(p_{z}=0\right)$ through the retrieved $3 \mathrm{D}$ momentum distribution for b) $\mathrm{D}_{2}^{+}$ions. c) Idem, for $\mathrm{D}^{+}$ions. d) The $\mathrm{D}^{+}$kinetic energy spectrum, resulting from an angular integration. The momentum distribution for the $\mathrm{D}^{+}$ ions is maximal at $E_{\mathrm{k}}=6 \mathrm{eV}$.

Figure 5. a) A slice $\left(p_{z}=0\right)$ through the $3 \mathrm{D}$ momentum distribution for the electrons resulting from ionization of $\mathrm{O}_{2}$. b) The corresponding electron kinetic energy spectrum, from an angular integration of the momentum distribution. The electron kinetic energy spectrum resulting from an integration $\pm 5^{\circ}$ perpendicular to the laser polarization axis is shown in red and shows additional peaks not resolved in the fully angle-integrated blue spectrum. c) A slice $\left(p_{z}=0\right)$ through the $3 \mathrm{D}$ momentum distribution for $\mathrm{O}^{+}$ions resulting from ionization of $\mathrm{O}_{2}$. d) The corresponding $\mathrm{O}^{+}$ion kinetic energy spectrum.

Figure 6. a) A slice $\left(p_{z}=0\right)$ through the $3 \mathrm{D}$ momentum distribution for electrons resulting from ionization of $\mathrm{N}_{2}$. b) The corresponding electron kinetic energy spectrum, resulting from an angular integration. The inset in red, is a result from an integration $\pm 10^{\circ}$ along the laser polarization axis and shows that the highest intensity peak actually consists of three peaks. The lower kinetic energy region was scaled by a factor to show the peaks more clearly. c) A slice $\left(p_{z}=0\right)$ through the $\mathrm{N}^{+} 3 \mathrm{D}$ momentum distribution. d) The corresponding $\mathrm{N}^{+}$kinetic energy spectrum, resulting from an angular integration of the momentum distribution

Figure 7. a) A slice $\left(p_{z}=0\right)$ through the $3 \mathrm{D}$ momentum distribution for electrons resulting from ionization of $\mathrm{CO}_{2}$. b) The corresponding electron kinetic energy spectrum. The peak in red, resulted from an integration $\pm 5^{\circ}$ along the laser polarization axis and shows that the second peak at $E_{\mathrm{k}}=27.74 \mathrm{eV}$ has an asymmetric feature at $E_{\mathrm{k}}=26.31 \mathrm{eV}$, not observed in the fully angle-integrated blue spectrum. The lower kinetic energy region was scaled with a factor to show the features more clearly. c), e), g) A slice $\left(p_{z}=0\right)$ through the 3D momentum distributions of $\mathrm{CO}^{+}, \mathrm{O}^{+}$and $\mathrm{C}^{+}$ions respectively. The corresponding ion kinetic energy spectra, from angular integration of the momentum distributions, are shown in $\mathrm{d}), \mathrm{f}$ ) and $\mathrm{h}$ ). 


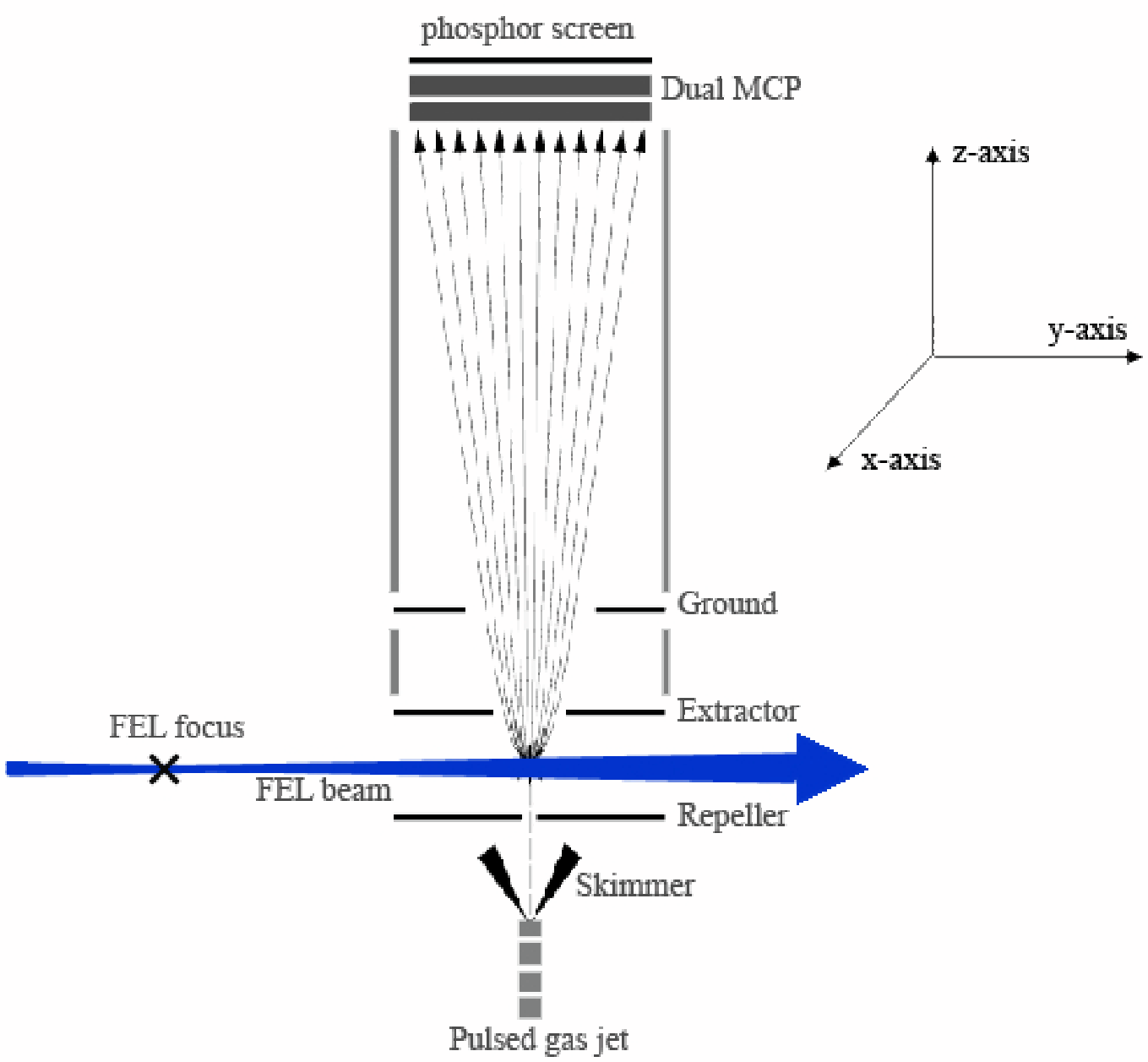

Figure 1: P. Johnsson et al., Experimental set-up 

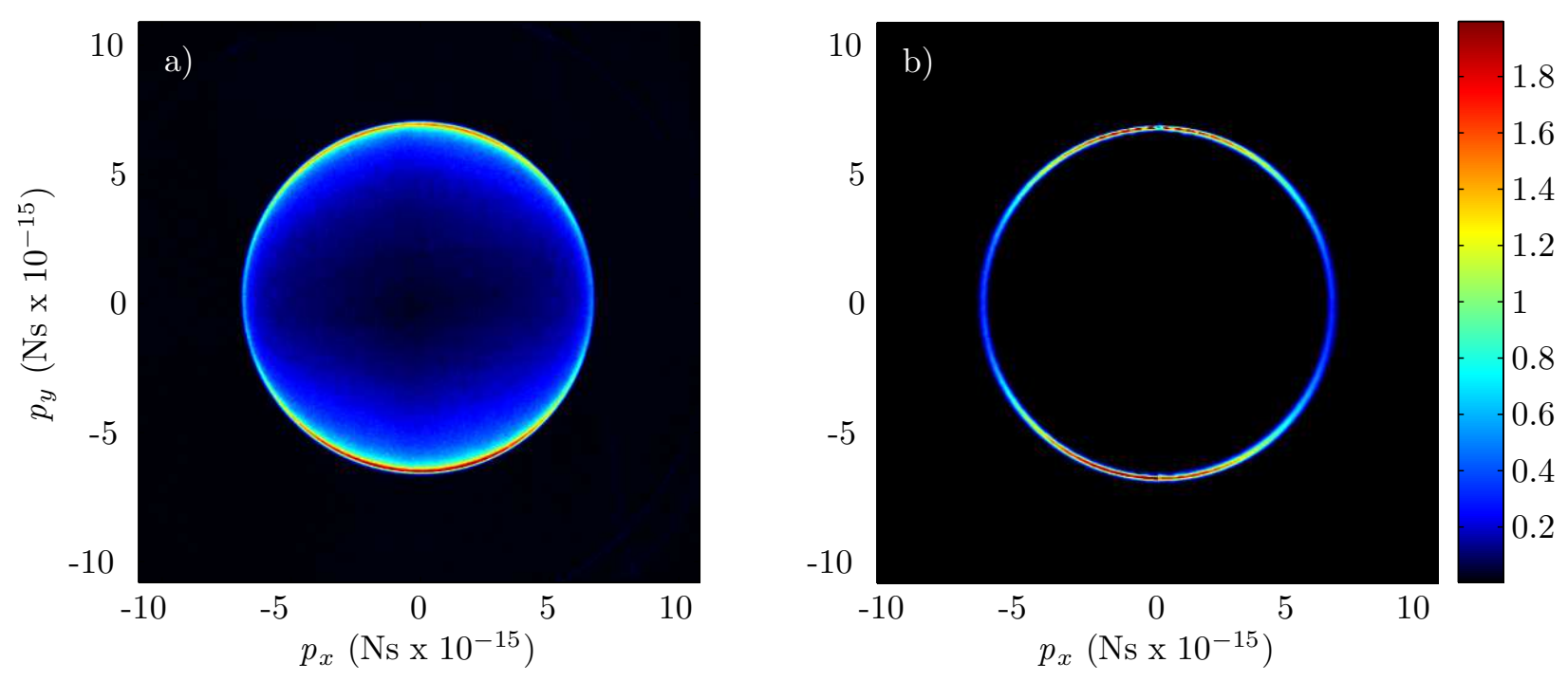

Figure 2: P. Johnsson et al., raw and inverted images
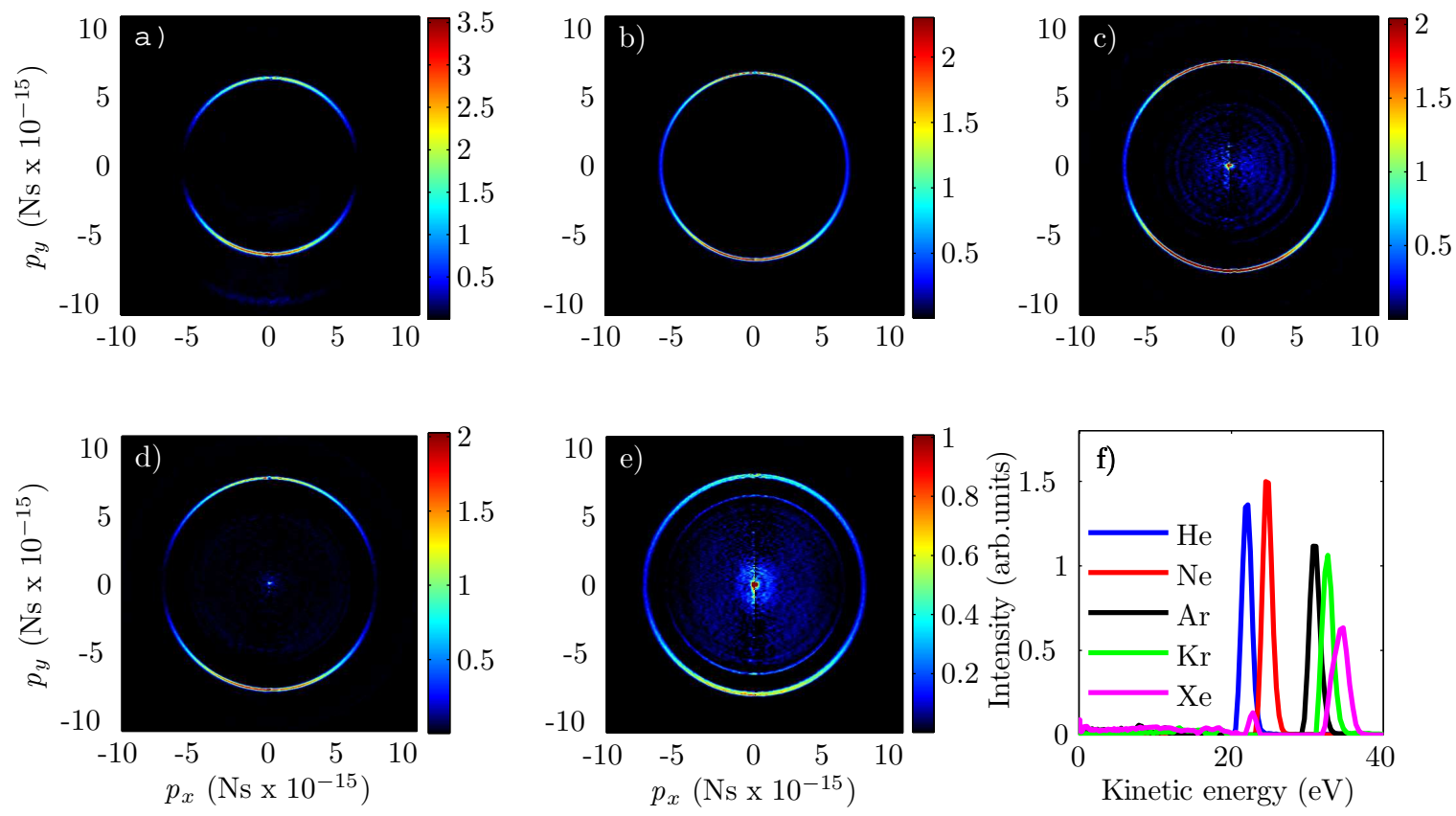

Figure 3: P. Johnsson et al., rare gases 

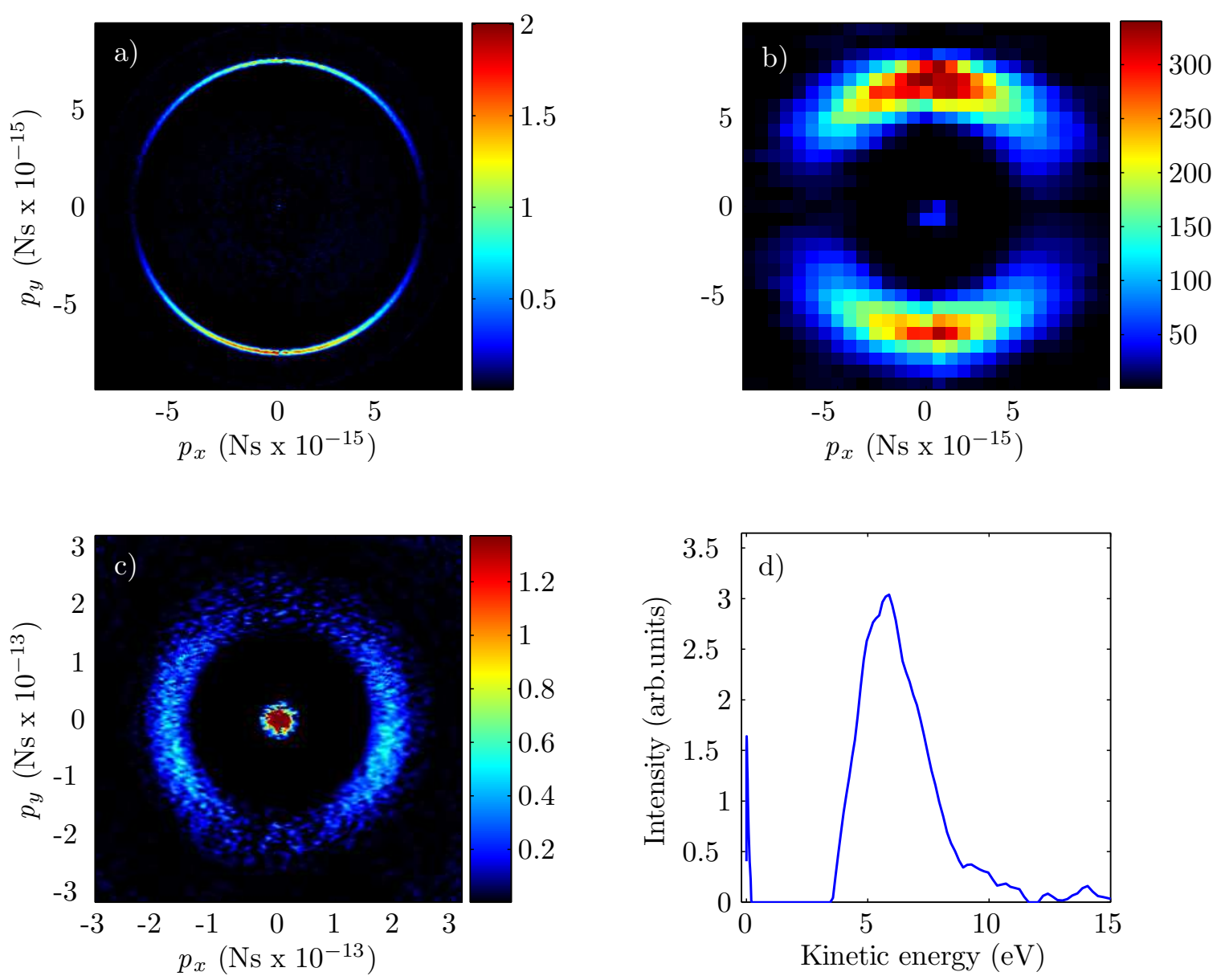

Figure 4: P. Johnsson et al., $\mathrm{D}_{2}$ 

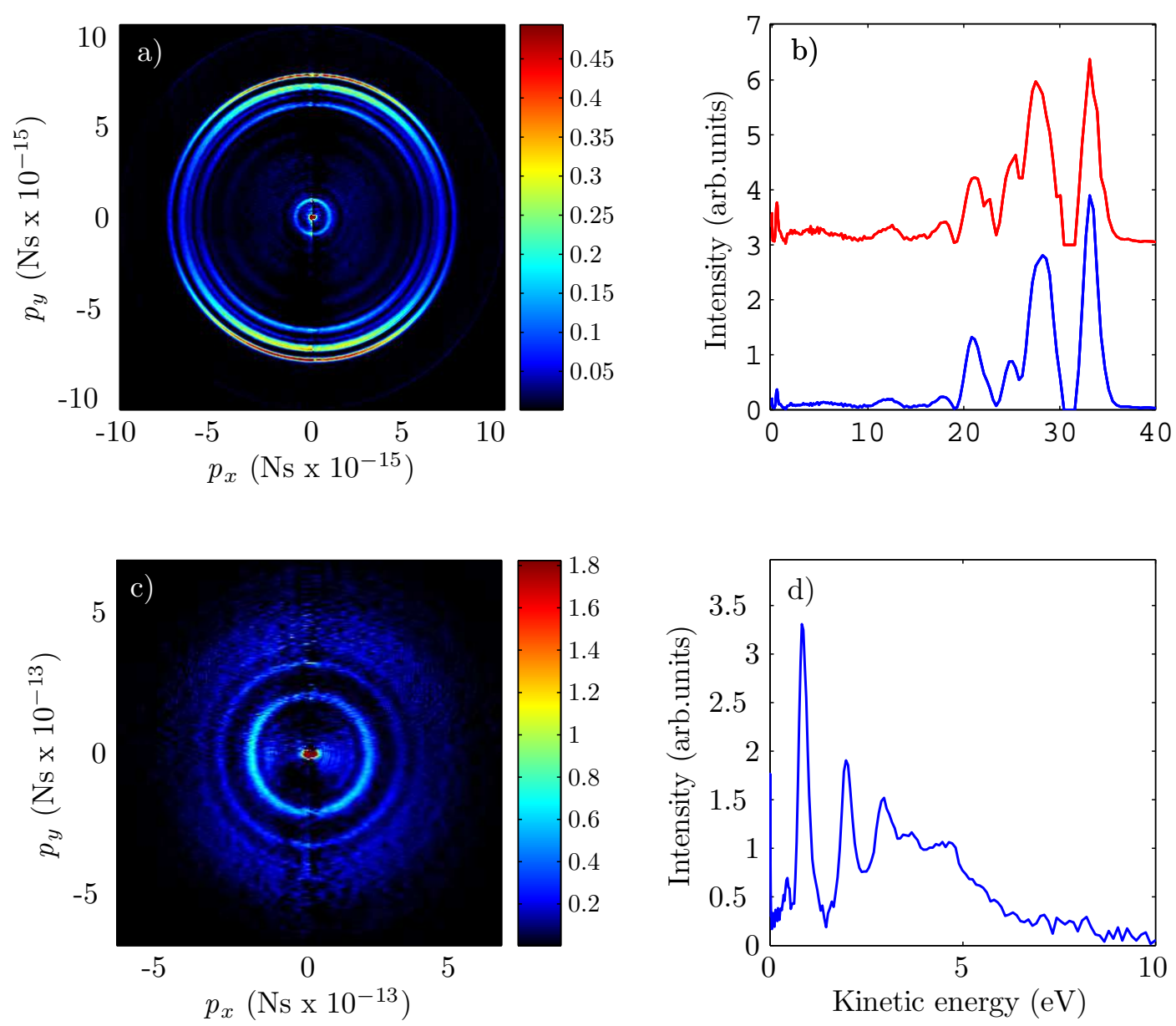

Figure 5: P. Johnsson et al., O2 

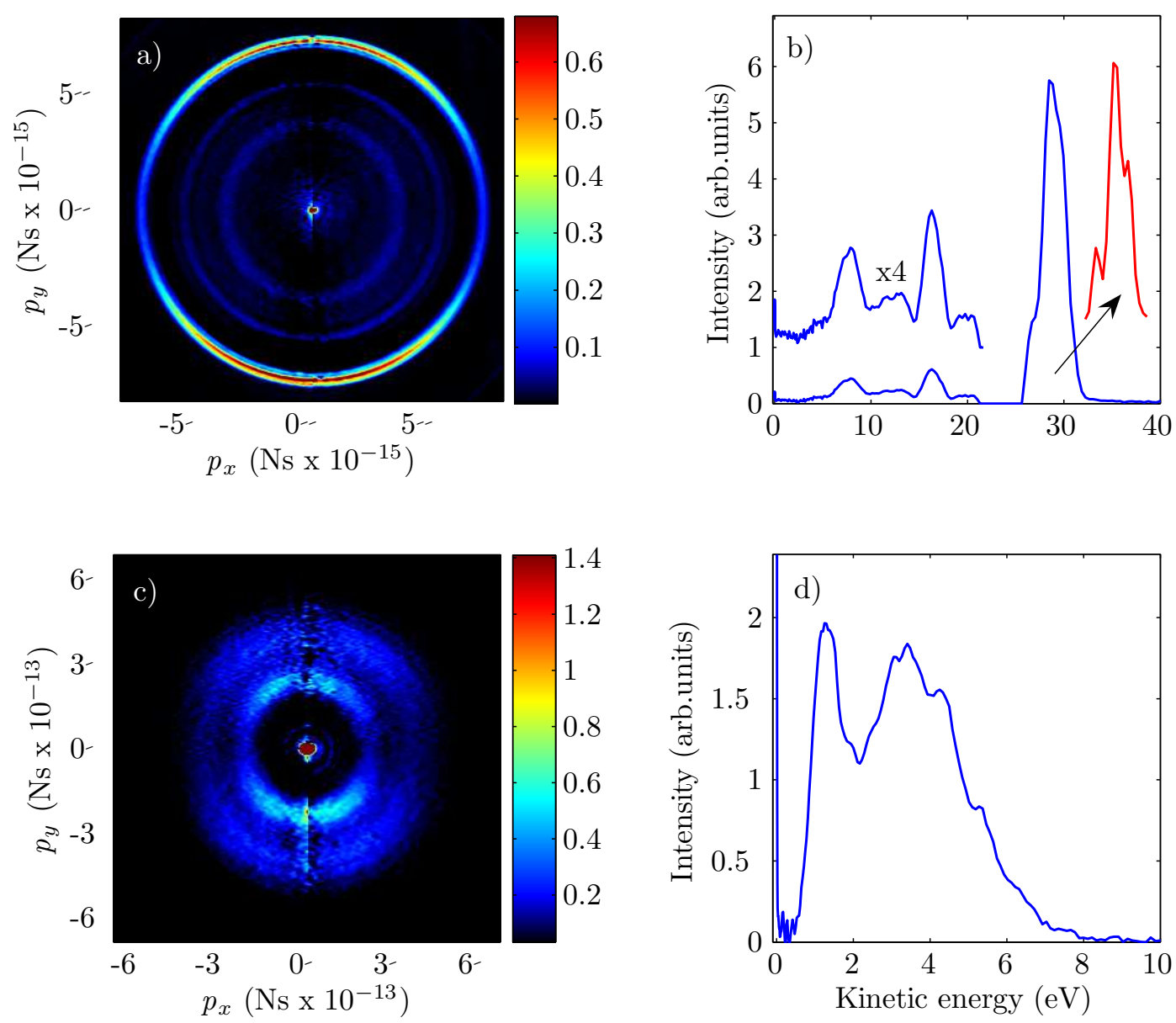

Figure 6: P. Johnsson et al., N2 

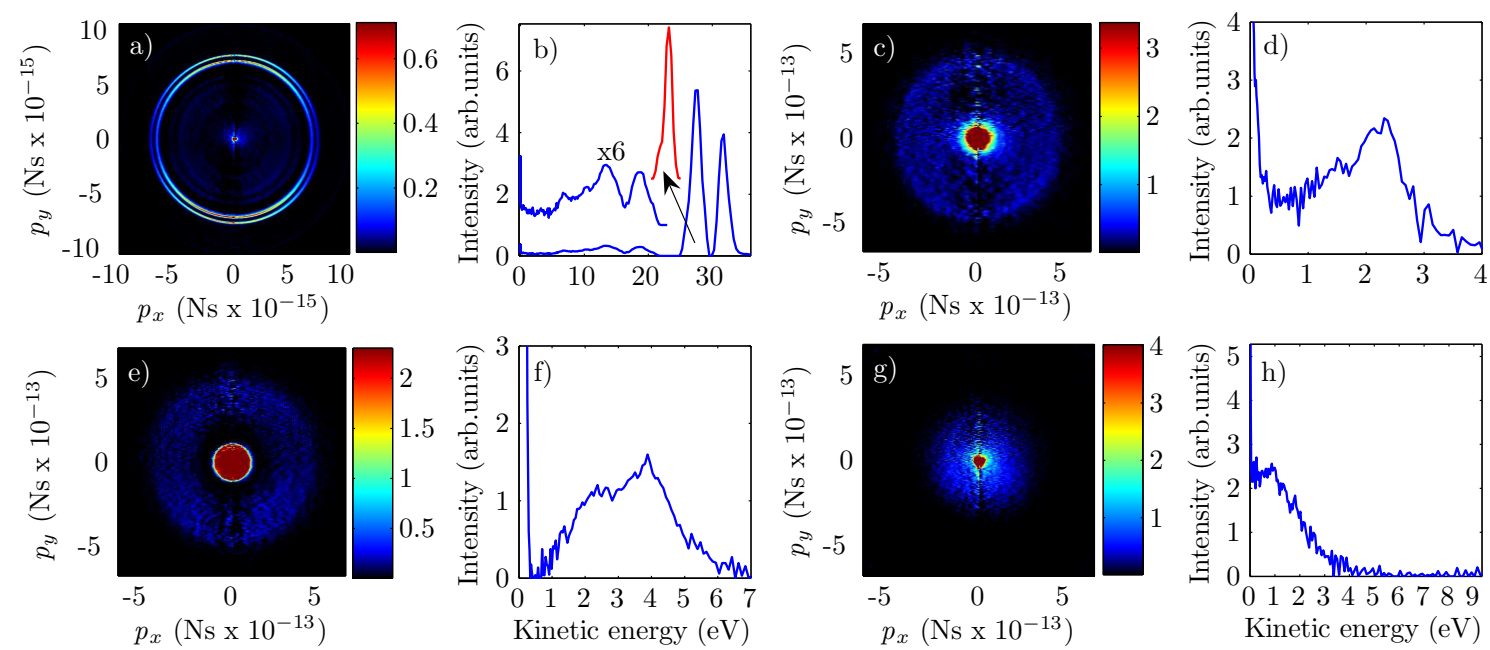

Figure 7: P. Johnsson et al., CO2 